\title{
Profile of Tofacitinib in the Treatment of Ulcerative Colitis: An Evidence-Based Review of Recent Data
}

This article was published in the following Dove Press journal:

Drug Design, Development and Therapy

\author{
Fumi Varyani ${ }^{1}, *$ \\ Konstantinos Argyriou (D) ${ }^{2, *}$ \\ Frank Phillips ${ }^{1} *$ \\ Eirini Tsakiridou ${ }^{3}$ \\ Gordon William Moran ${ }^{2,4}$ \\ 'Queen's Medical Center, Nottingham \\ University Hospitals NHS Trust, \\ Nottingham, UK; ${ }^{2}$ Nottingham Digestive \\ Diseases Centre, School of Medicine, \\ University of Nottingham, Nottingham, \\ UK; ${ }^{3}$ University Hospital of Athens \\ "LAIKO", Athens, Greece; ${ }^{4}$ National \\ Institute of Health Research, Nottingham \\ Biomedical Research Centre at \\ Nottingham University Hospitals \\ National Health Service Trust and the \\ University of Nottingham, Nottingham, \\ UK
}

*These authors contributed equally to this work
Correspondence: Konstantinos Argyriou Nottingham Digestive Diseases Centre, Room W/E West Block, Queen's Medical Centre, Nottingham NG7 2UH, UK Email kosnar2@yahoo.gr

\begin{abstract}
Recent advances in the understanding of the pathophysiology of ulcerative colitis (UC) have led to the expansion of our therapeutic arsenal. Conventional treatment options, including aminosalicylates, corticosteroids, thiopurines, and calcineurin inhibitors, fail to control the disease in a significant proportion of patients. Approximately $25-50 \%$ of the patients treated with tumor necrosis factor antibodies (anti-TNF $\alpha$ ) are primary and secondary non-responders to therapy. Tofacitinib is a novel orally administered small synthetic molecule that inhibits a homologous family of enzymes, termed Janus kinases that modulate multiple key cytokines involved in the pathogenesis of UC. Phase II and III trials showed promising results in UC, leading the Food and Drug Administration (FDA) and the European Medicines Agency (EMA) to approve its administration for the induction and maintenance of remission in moderate-to-severe UC. Herein, we review tofacitinib for the management of $\mathrm{UC}$, its mechanism of action pharmacokinetic properties, efficacy, and safety.
\end{abstract}

Keywords: tofacitinib, ulcerative colitis, Janus kinase inhibitors, Jakinibs

\section{Introduction}

Ulcerative colitis (UC) is an idiopathic immune-mediated inflammatory bowel disease of the colon. ${ }^{1}$ It is often diagnosed in the third and fourth decades of life ${ }^{2}$ and leads to significant mortality and morbidity. ${ }^{3}$ Both UC and Crohn's disease are associated with economic impact from hospitalization and surgery and also loss of productivity at work. ${ }^{3,4}$

More than $80 \%$ of the UC patients run a relapsing disease course, whereas $10-15 \%$ experience an aggressive course. ${ }^{5,6}$ In $30 \%$ of the patients, the disease distribution is left-sided. Symptoms of UC include diarrhea, rectal bleeding, urgency, tenesmus, and fecal incontinence. Patients with UC are at risk of dysplasia and cancer. Between 10\% and $15 \%$ undergo colectomy to manage disease or its complications. ${ }^{7}$

Current medical treatment includes aminosalicylates, corticosteroids, thiopurines, and calcineurin inhibitors. Anti-TNF $\alpha$ antibodies ${ }^{8-11}$ are available, but contraindications, primary non-response, loss of response, and intolerance often occur.

In addition to TNF $\alpha$, there are several other cytokine pathways involved in the development of UC. ${ }^{12}$ The IL-23/IL-17 axis is important in human IBD, with elevated Th17 cytokines in human IBD. ${ }^{13}$ TGF $\beta$-deficient mice develop severe multiorgan lymphoproliferative disease. ${ }^{14}$ IL-10-deficient mice develop spontaneous colitis. $^{15}$ The discovery of specific pathways involved in IBD has led to more specific medications targeting these, e.g., ustekinumab targeting the p40 subunit shared between IL12 and IL23. UC is, however, most likely a heterogeneous condition, 
with multiple different immune pathways involved in an individual resulting in a common phenotype - therefore, suppression of more than one cytokine pathway may be beneficial.

Tofacitinib (XELJANZ/XELJANZ XR; Pfizer, NY, USA) is an oral small molecule pan-Janus kinase (JAK) inhibitor (molecular weight $312.4 \mathrm{Da} ; 504.5$ for the citrate salt). This is the first oral formulation for the treatment of UC; tofacitinib is less immunogenic than the larger biologic antibodies, has a shorter half life, and has therefore got the advantage of allowing rapid discontinuation in cases of adverse events. ${ }^{16}$

The JAKs are the downstream signaling molecules of a large number of the cytokine pathways involved in IBD. ${ }^{17}$ When a cytokine binds its cell surface receptors, it dimerizes the ligand receptors and this results in phosphorylation of the JAK molecules. JAK then activates signal transducer and activator of transcription molecules (STATs), the phosphorylation of which results in STAT migration to the nucleus and activation of gene transcription. ${ }^{17}$ By targeting JAK signaling, it is possible to affect multiple cytokine pathways thought to be involved in colitis.

Tofacitinib had been initially approved for the treatment of moderate-to-severe rheumatoid arthritis (RA) [2012] and psoriatic arthritis (PsA) [2017] in adult patients with inadequate response or intolerance to prior disease-modifying antirheumatic drug therapies. ${ }^{18}$ Phase II and phase III trials showed promising results in UC, leading the Food and Drug Administration (FDA) and the European Medicines Agency (EMA) to approve tofacitinib for the induction and maintenance of remission in moderate-to-severe UC. ${ }^{19,20}$ For induction, $10 \mathrm{mg}$ is given orally twice daily for 8-week period, followed by a maintenance dose of $5 \mathrm{mg}$ twice daily. ${ }^{19,20}$ Nevertheless, in case of inadequate response by week 8 , the dose of $10 \mathrm{mg}$ can be extended for another 8 weeks (16 weeks total) prior to attempting dose reduction or can be maintained at $10 \mathrm{mg}$ in those patients who had previously failed anti-TNF $\alpha .{ }^{19}$ We review the pharmacokinetic properties, efficacy, and safety of tofacitinib in UC.

\section{Search Strategy}

Medline, PubMed, and Scopus search were undertaken by two of the authors (G.M and K.A). The search criteria used were "JAK inhibitor", "tofacitinib”, "CP-690 550", "Ulcerative colitis", "pharmacokinetic", "side effects", "adverse events", and "complications". A manual search of the clinical trial register, article reference lists, abstracts from Digestive Disease Week, United European Gastroenterology Week, and European Crohn's Colitis Organization congresses were also conducted up to the 17th of May 2019. After an initial title screen, each relevant article was subsequently reviewed.

\section{Overview of JAKs}

JAKs compose a family of tyrosine kinases, that includes four large peptide members $(\geq 1100$ amino acids/molecular mass: 120-140 kilodaltons), named JAK 1, JAK 2, JAK 3, and Tyrosine Kinase 2 (TYK-2). Of the four family members, JAK1, JAK2, and TyK2 are ubiquitously expressed, whereas JAK3 is preferentially expressed in hematopoietic cells. ${ }^{17,21}$ All family members have an evolutionary conserved structure, which encompasses the catalytically active and inactive functions of the enzymes and are associated with the different chains of type I/II cytokine receptors intracellularly ${ }^{21,22}$ A large group of cytokines, including those involved in the pathogenesis of UC, employ type I and II transmembrane receptors that rely on JAKs for intracellular signaling. ${ }^{17}$ JAK1 and JAK3 are downstream of cytokine receptors containing the IL-2 receptor common $\gamma$ chain (e.g., IL-2, IL-4, IL-7, IL-9, IL-15, IL-21), while JAK2 transduce signals from the cytokines binding to hematopoietic growth factor and gp40-containing cytokine receptors. At least two homologous (e.g., JAK2/JAK2) or heterologous (e.g., JAK1/JAK3) JAK molecules are required from each receptor in order to signal. Cytokine binding to the receptor leads to JAK activation, auto-phosphorylation, and phosphorylation. This interaction forms specific binding sites, recruits one or more members of a family of transcription factors, termed STAT (Signal Transducer and Activator of Transcription) proteins, and induces their subsequent phosphorylation. ${ }^{17}$ Once activated, STATs dissociate from the receptor and form homo-or hetero-dimers and translocate to the nucleus, where they bind to DNA sequences, and influence gene expression ${ }^{17,21}$ as demonstrated in Figure 1.

JAKs regulate a variety of cytokine-mediated processes including hematopoiesis, inflammation, innate, and adaptive immunity. Aberrant activation of JAKs peptides has been shown to lead either directly or indirectly to a variety of autoimmune and inflammatory disorders, including UC. ${ }^{23}$ Hence, it is of no surprise that the pharmacological inhibition of JAK has gained significant attention as a therapeutic target in immune-mediated disorders, with recent genomewide association studies supporting the rationale of targeting JAKs in UC. ${ }^{23,24}$ The JAK cytokines, however, are not responsible for all cytokine signaling. For example, TNF $\alpha$ mediates gene expression through the NF-kB, JUN kinase, p38 MAPK, and ERK1/ERK2 pathways. $^{25}$ 


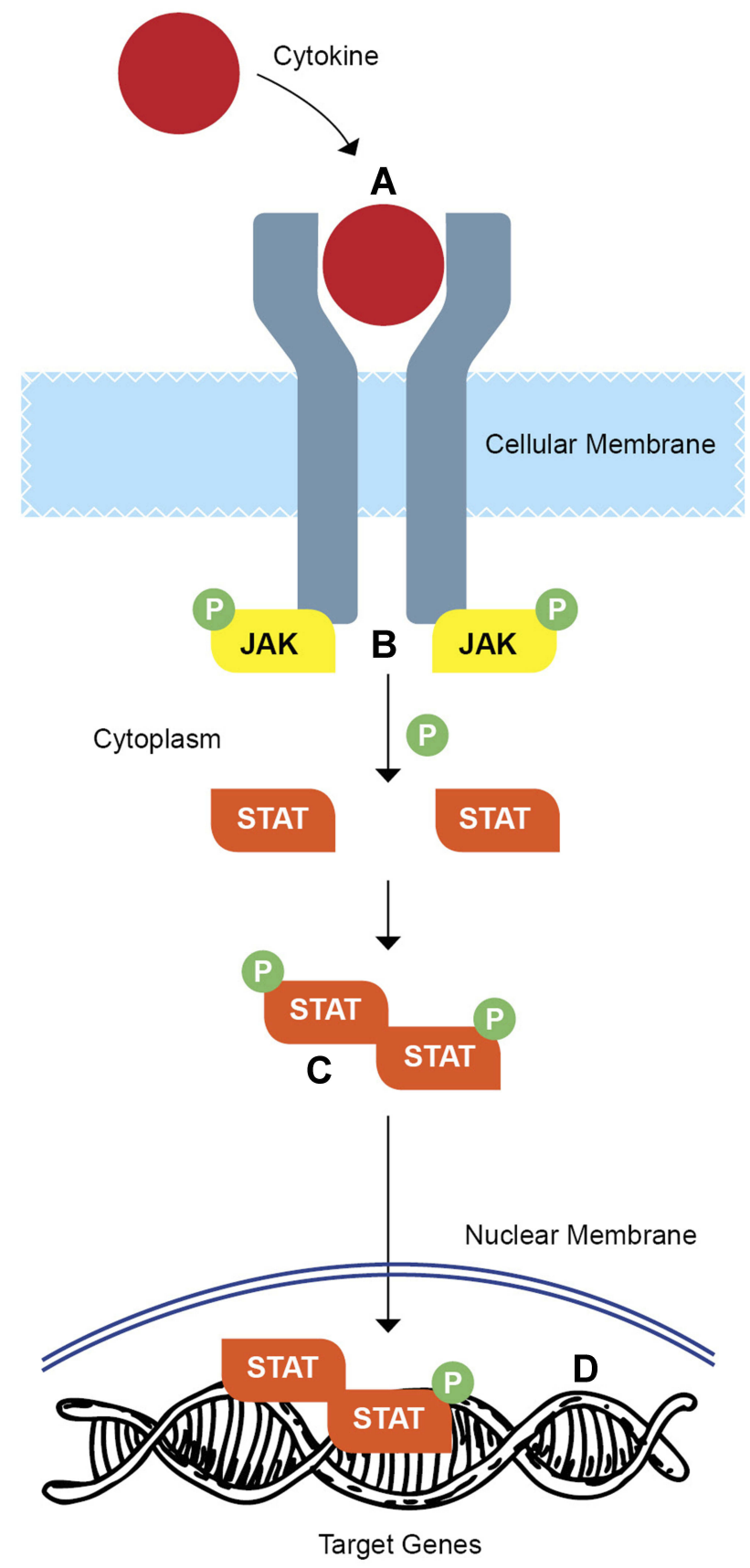

Figure I Overview of the mechanism of action of Janus kinases (JAKs). Cytokine binding to type I/II receptor at the cytoplasmic side of the plasma membrane leads to JAK activation and phosphorylation. (A) This subsequently initiates recruitment of two signal transducer and activator of transcription proteins (STATs) and induces their subsequent phosphorylation (B). Once phosphorylated, STAT homodimerize or heterodimerize (C) and translocate to the nucleus where they regulate gene transcription (D).

\section{JAK Inhibition and Tofacitinib: Mechanism of Action}

Tofacitinib (CP-690, 550) was one of the first JAK inhibitors investigated. After demonstrating significant immunosuppressive effects in pre-clinical animal models of transplantation $^{26,27}$ and arthritis, ${ }^{28}$ tofacitinib entered clinical trials in patients with RA. Tofacitinib has undergone further assessment in various other immune-mediated conditions, including PsA and UC. ${ }^{29}$

In order to exert its pharmacologic action, tofacitinib diffuses intracellularly, and then binds to the adenosine triphosphate (ATP) binding site in the catalytic cleft of the kinase domain of JAKs. This action inhibits JAK's subsequent phosphorylation and STAT activation, ${ }^{30}$ and attenuates downstream cytokine production with a consequent downregulation of the immune response. ${ }^{31}$

Tofacitinib is a pan-JAK inhibitor. ${ }^{28}$ Although in vitro studies suggested that tofacitinib inhibits JAK1, JAK2, JAK3, and to a lesser extent TYK2 in human cells, in vivo studies have demonstrated it preferentially inhibits JAK1 and JAK3 pathways. ${ }^{28}$ As a result, tofacitinib was shown to disrupt to greater extent signaling from JAK3and JAK1-dependent cytokines (e.g., IL-2, IL-6, interferons [IFN], IL-12, IL-4, IL-7, IL-15, and IL-21), affecting lymphocyte activation, proliferation, and function that are crucial for multiple aspects of the immuno-inflammatory response observed in $\mathrm{UC}^{27,28,32}$

\section{Pharmacokinetic Profile}

The pharmacokinetic (PK) profile of tofacitinib has been studied in healthy volunteers and in patients with RA, PsA, and UC.

In healthy volunteers, tofacitinib's time to serum peak concentrations is between 0.5 and $1 \mathrm{hrs}$, and it is eliminated in $3 \mathrm{hrs}$. No correlation was observed between dosage and lymphocyte subsets in healthy volunteers. ${ }^{33}$ In UC, plasma tofacitinib concentrations increased proportionally with dose and steady-state levels did not change between baseline and 8 weeks. Steady-state concentrations did not differ according to disease activity ${ }^{34}$ neither in UC nor in psoriatic arthropathy. ${ }^{35}$

The majority ( $70 \%$ ) of the drug is cleared by the liver, with $30 \%$ being renally excreted. ${ }^{16}$ The clearance of tofacitinib is mediated by cytochrome P450 (CYP) 3A4; however, it does not affect the clearance of other drugs metabolized by CYP enzymes. ${ }^{36}$ Use of other drugs that inhibit CYP 34A systems such as fluconazole and ketoconazole increased the half-life of tofacitinib by an hour. This would suggest that some dosage adjustment is required in the context of concomitant CYP34A inhibitor usage. ${ }^{37} \mathrm{CrCl}$ also affects the half-life of tofacitinib. ${ }^{35}$

Population PK analysis from 1096 UC patients with moderate-to-severe disease activity, pooled across one phase 2 and three phase 3 studies, revealed comparable 
PK properties for tofacitinib with those observed in other immune-mediated conditions such as PsA and RA. Based on this comparability, all proposed dosing adjustment recommendations derived from phase 1 studies in $\mathrm{RA}$ are also applicable to UC patients. Consequently, in UC patients with moderate renal (defined as creatinine clearance values less than $60 \mathrm{~mL} / \mathrm{min}$ ), it is suggested to reduce to dose of tofacitinib by half. In contrast, no dose modification has been shown to be required for tofacitinib in association with a variety of other patientand disease-related factors including age, body weight, sex, ethnicity, and baseline disease severity (e.g., baseline Mayo score and baseline albumin). Tofacitinib has not been studied in the context of severe hepatic impairment. $^{19}$

\section{Therapeutic Efficacy of Tofacitinib in UC}

A summary of the market-authorizing trials of tofacitinib's in UC included in this review is shown in Table 1.

\section{Phase II Studies}

Sandborn et al investigated tofacitinib in a multi-center, double-blinded, randomized controlled trial over an 8-week period for moderate-to-severe UC. ${ }^{38}$ Patients were assigned to placebo administered twice daily or tofacitinib (at doses of $0.5,310$, or $15 \mathrm{mg}$ twice daily). The primary endpoint was clinical response (a decrease from baseline total Mayo score by at least 3 points and a relative decrease by at least $30 \%$ ) at the end of the 8 -week period. The secondary endpoint was clinical remission defined as a total Mayo score of 0 to 2 .

At 8 weeks, clinical response was observed in $32 \%$, $48 \%, 61 \%$, and $78 \%$ of the patients receiving tofacitinib at doses $0.5,3,10$, and $15 \mathrm{mg} \mathrm{BD}$, respectively ( $\mathrm{p}$ values $0.39,0.55,0.10$, and $<0.001$ ), compared with $42 \%$ of the patients receiving placebo. Endoscopic response was observed in $52 \%, 58 \%, 67 \%$, and $78 \%$ of the patients on tofacitinib at doses of $0.5,3,10$ and $15 \mathrm{mg} \mathrm{BD}$, respectively, compared to $46 \%$ of the placebo ( $p$ values 0.64 , $0.30,0.07$, and 0.001).

Although its highest efficacy was shown to be expected from doses $\geq 10 \mathrm{mg}$ twice daily, safety concerns (dosedependent hyperlipidemia and viral infections) that have arisen from patients with RA receiving more than $15 \mathrm{mg}$ $\mathrm{BD}^{39}$ has led the FDA to select the $10 \mathrm{mg}$ dose for the subsequent clinical development of tofacitinib in UC. ${ }^{38}$

\section{Efficacy in Phase III Trials}

Induction of Remission and Mucosal Healing

The efficacy of tofacitinib in the induction of remission in patients with moderately-to-severely active UC was investigated in two multi-center, double-blind, placebocontrolled phase 3 trials, named OCTAVE (Oral Clinical Trials for tofAcitinib in ulceratiVE colitis) Induction 1 and $2 .^{40}$ In these trials, 1139 eligible patients were randomized (4:1) to receive tofacitinib $10 \mathrm{mg}$ or placebo twice a day for an 8-week period. The patients had moderately-tosevere active (mean total Mayo score: 9) extensive colitis $(52 \%)$ with a median disease duration of 6 years, mean age of 41 years, with $59 \%$ of the participants being male and $80 \%$ caucasian. Approximately $70 \%$ of the patients had previously failed immunosuppressants other than biologic agents or corticosteroids, $52 \%$ had failed treatment with anti-TNF $\alpha$ (56\% primary non-responders), and $46 \%$ were taking oral corticosteroids upon entry. Aminosalicylates or prednisolone up to $25 \mathrm{mg}$ OD was allowed over the treatment period, but no immunomodulators or biologic therapies. The primary endpoint was clinical remission at 8 weeks (defined as a total Mayo score $\leq 2$ points, no individual subscore $>1$, rectal bleeding subscore of 0 ). The key secondary endpoint was mucosal healing ( $\mathrm{MH}$ defined as endoscopic subscore of 0 or 1) at 8 weeks. Clinical remission at 8 weeks occurred in $19 \%$ and $17 \%$ of the tofacitinib group, whereas the placebo groups had a remission rate of $8 \%$ and $4 \%$ in OCTAVE Induction 1 and 2, respectively (both comparisons were statistically significant).

MH was achieved in 31\% (OCTAVE 1) and 28\% (OCTAVE 2) in tofacitinib versus $16 \%$ and $12 \%$ in those receiving placebo $(\mathrm{p}<0.001)$.

In both induction trials, subgroup analysis showed no significant differences in the efficacy of TOF over placebo for ethnic origin, previous experience to anti-TNF $\alpha$ and concomitant use of aminosalicylates. ${ }^{40,41}$ At all visits, patients receiving TOF had larger improvement in partial Mayo score compared to placebo with significant reductions in stool frequency. ${ }^{42}$

\section{Maintenance of Remission and Mucosal Healing}

A total of 593 patients from the OCTAVE Induction 1 or 2 trial who achieved clinical response at the end of 8 weeks with TOF $10 \mathrm{mg}$ twice daily were re-randomized, in a 1:1:1 ratio into OCTAVE Sustain. These patients received maintenance therapy with tofacitinib 5 or $10 \mathrm{mg}$ twice daily or placebo for 52 weeks. In this trial, $30 \%$ of the participants were in remission upon entry. The primary endpoint of 


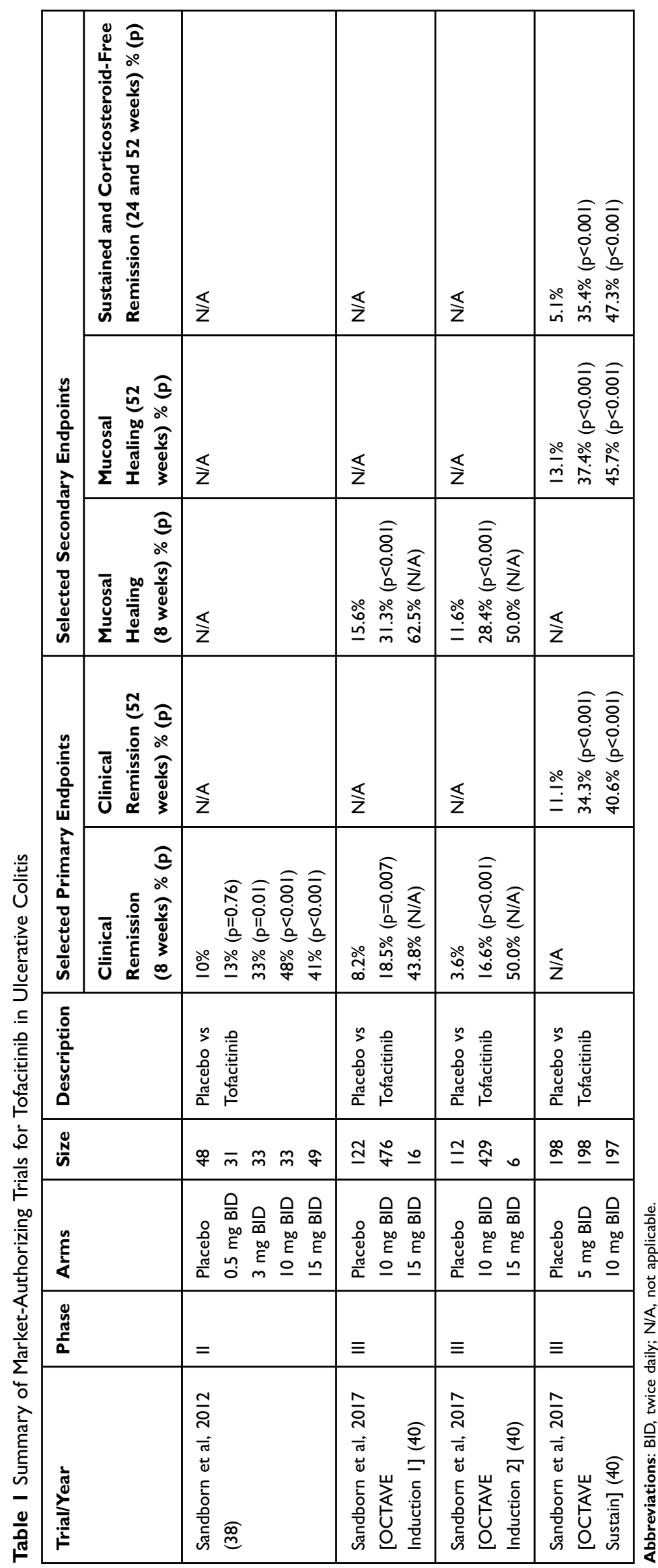


OCTAVE Sustain was remission at 52 weeks. Key secondary endpoints were the achievement of $\mathrm{MH}$ at 52 weeks and of sustained glucocorticoid-free remission defined as glucocorticoid-free remission at 24 and 52 weeks. At 52 weeks, 34\% of the $5 \mathrm{mg}$ tofacitinib group, $41 \%$ in the tofacitinib $10 \mathrm{mg}$ group, and $11 \%$ in the placebo group were in remission. As high as $37 \%$ in the patients receiving tofacitinib $5 \mathrm{mg}, 46 \%$ of the tofacitinib receiving $10 \mathrm{mg}$, and in $13 \%$ of those receiving placebo had achieved MH. With regard to the other key efficacy endpoints, sustained and glucocorticoid-free remission were achieved by $35 \%$ and $47 \%$ of the $5-\mathrm{mg}$ and $10-\mathrm{mg}$ tofacitinib treatment arms as compared with the placebo arm, respectively $(5 \%, \mathrm{p}<0.001$ for both comparisons $) .{ }^{40}$

In subsequent subgroup analysis, no significant differences in efficacy of tofacitinib over placebo with regard to patients' race and concomitant use of aminosalicylates ${ }^{40,41,43}$ were noted. In addition, irrespective of the tofacitinib dose received or anti-TNF $\alpha$ failure status, patients who entered the trial in remission were more likely to remain in remission or $\mathrm{MH}$ at week 52, compared to those who had not achieved remission after induction. ${ }^{44}$ At all visits, patients receiving tofacitinib had better improvements in the partial Mayo score as compared to those receiving placebo $(\mathrm{p}<0.001$ for all comparisons). ${ }^{40,41}$

\section{Long-Term Efficacy}

The long-term efficacy of tofacitinib is being evaluated in an ongoing, open-label, long-term extension (OLE) study, named OCTAVE Open. In OCTAVE Open, all patients who had completed or experienced treatment failure in OCTAVE Sustain as well as non-responders to tofacitinib from OCTAVE Induction 1 and 2 were included (Figure 2). The preliminary results of this study are summarized below.

\section{Recapturing Response}

Loss of Response Secondary to Treatment Interruption The effect of tofacitinib in recapturing response after treatment interruption was studied in a subpopulation of 101 patients entered into the OCTAVE Open trial. These patients had achieved clinical response with tofacitinib $10 \mathrm{mg}$ twice daily in the OCTAVE Induction trials, but experienced treatment failure - loss of response - in the maintenance study after being randomized to receive placebo. Treatment failure was defined as increase $\geq 3$ points from OCTAVE Sustain baseline total Mayo score plus increase in rectal bleeding subscore and endoscopic subscore $\geq 1$ point and absolute endoscopic subscore $\geq 2$ points after $\geq 8$ weeks of maintenance therapy. All of these patients received tofacitinib $10 \mathrm{mg}$ twice daily in the OLE study. Study outcomes included clinical response, $\mathrm{MH}$, and remission at months 2 and 12 of OCTAVE Open. At month 2, 76\%, 55\%, and 40\% of the subpopulation achieved clinical response, $\mathrm{MH}$ and clinical remission, respectively. Similar findings were identified at 12 months in these key outcomes. Based on their findings, Panes et al suggested that in tofacitinib responders who lost response secondary to treatment interruption

OCTAVE Induction 1

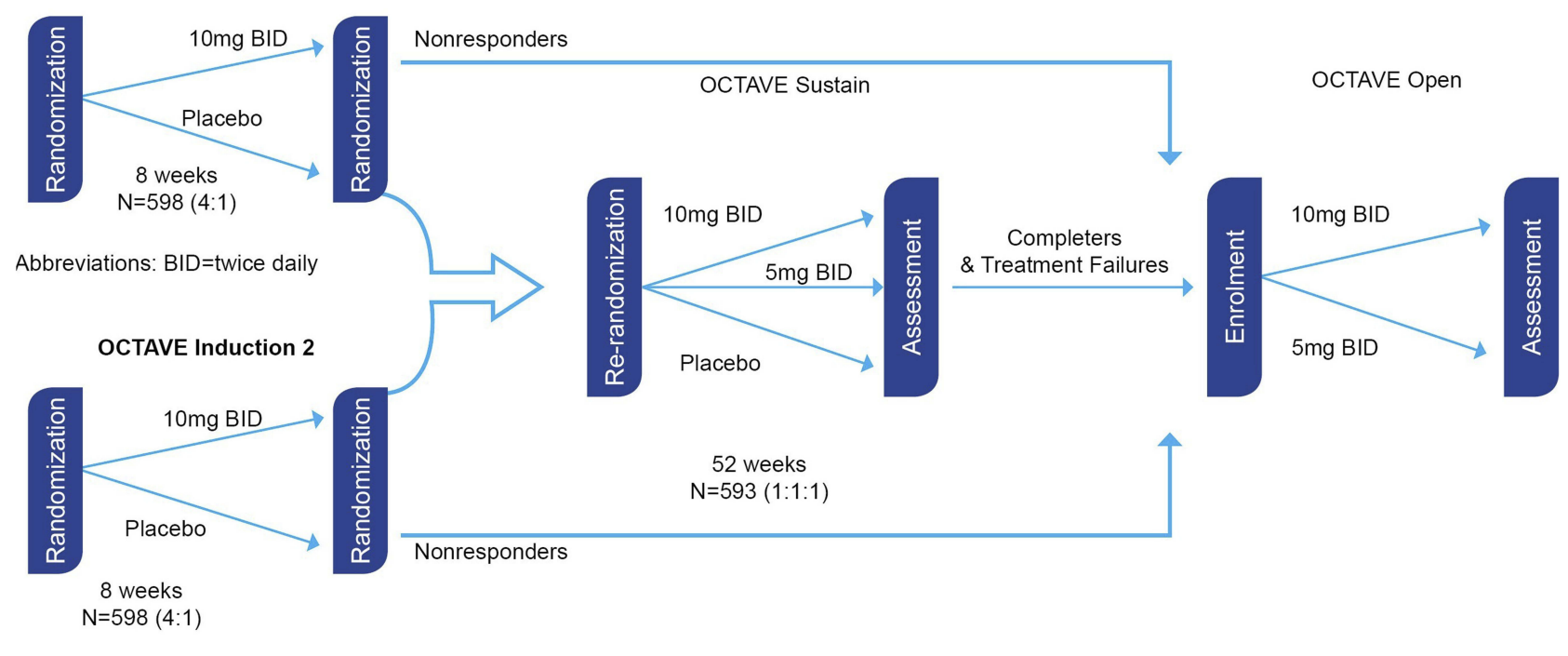

Abbreviations: $B I D=t w i c e$ daily

Figure 2 Flowchart of participants progress through the OCTAVE trials. 
retreatment with $10 \mathrm{mg}$ twice daily, recaptures response in approximately three-quarters of patients within 2 months and sustains the response up to 1 year. $^{45}$

\section{Loss of Response Secondary to Dose Reduction}

Tofacitinib to recapture response after dose reduction was investigated in a subpopulation of 58 patients who had initially responded to tofacitinib in the OCTAVE Induction trials but lost response in the maintenance study after being allocated to tofacitinib $5 \mathrm{mg}$ twice daily. All of these patients were treated with tofacitinib10 $\mathrm{mg}$ twice daily in the OLE study. Study outcomes included clinical response, $\mathrm{MH}$, and remission at months 2 and 12. At month 2, 58.6\%, $41.4 \%$, and $34.5 \%$ of the patients achieved clinical response, $\mathrm{MH}$, and clinical remission, respectively. Similar findings were identified at 12 months in these key outcomes $(68.8 \%, 60.4 \%$, and $52 \%$ for clinical response, $\mathrm{MH}$, and remission, respectively). Based on these data, Sands et al suggested that in tofacitinib responders who lost response secondary to dose reduction for maintenance, retreatment with $10 \mathrm{mg}$ twice daily recaptures response for most patients within 2 months following dose escalation. ${ }^{46}$

Induction of Response in Non-Responders to the Initial 8-Week Course (Delayed Response)

The efficacy of tofacitinib in inducing response in patients who failed to respond to an initial 8-week of tofacitinib $10 \mathrm{mg}$ twice daily was investigated in 295 patients from both OCTAVE Induction trials. All of these patients continued to receive tofacitinib $10 \mathrm{mg}$ twice daily in the OLE study. Study outcomes included clinical response, $\mathrm{MH}$, and clinical remission evaluated at months 2, 12, 24, and 36. At month 2, clinical response was observed in $51 \%$ of the subpopulation, with $72 \%, 61 \%$, and $54 \%$ of the patients preserving response at 12, 24, and 36 months, respectively. Approximately half of the patients were in remission $(49 \%, 45 \%$ and $45 \%$ at months $2,12,24$, and 36 , respectively), while $\mathrm{MH}$ was observed in the majority of patients $(57 \%, 53 \%$, and $51 \%$ at months $2,12,24$, and 36 , respectively) at any time-point after the first year of treatment. Similar trends over time were observed when prior failure to anti-TNF $\alpha$ was taken into consideration. Based on these findings, Rubin et al suggested that a substantial proportion of non-responders to the initial 8 -week course of tofacitinib at dose $10 \mathrm{mg}$ twice daily respond to an extended induction course and achieved response lasting up to 3 years, irrespective of patients' previous failure status to anti-TNF $\alpha^{47}$
Improvement of Remission After 52 Weeks of Response The efficacy of tofacitinib in achieving remission after prolonged response was investigated in a subpopulation of 82 patients who had responded to tofacitinib at 52 weeks. Of the 82 patients, 1 entered the Sustain trial in error, 18 were randomized to receive placebo, 28 to tofacitinib $5 \mathrm{mg}$ twice daily and 35 to tofacitinib $10 \mathrm{mg}$ twice daily. All of these patients received tofacitinib $10 \mathrm{mg}$ twice daily in the OCTAVE Open trial. Efficacy endpoints included clinical response, $\mathrm{MH}$, and clinical remission at months 2, 12, 24, and 36. At 24 months, clinical response was maintained by $70 \%$ of the patients. No difference in clinical response was observed between participants who had previously failed (65\%) or not failed (73\%) anti-TNF $\alpha$ therapy. By month 2, remission rates were improved in the majority of patients regardless of anti-TNF $\alpha$ failure status (59\% [48/82], 61\% [23/38] and 57\% [25/44] of the total number of patients and of those with and without failure to anti-TNF $\alpha$, respectively). Similar trends were observed with regard to $\mathrm{MH}$ over time. Based on these data, tofacitinib $10 \mathrm{mg}$ twice daily was shown to be effective in inducing remission in UC patients with prolonged response who had not achieved remission, regardless of their previous failure status to anti-TNF $\alpha{ }^{48}$

\section{Maintenance of Remission Following Dose Reduction}

The maintenance of remission for patients following a dose reduction in tofacitinib was investigated in a cohort of 76 patients from the SUSTAIN trial. ${ }^{49}$ All of these patients were in remission at 52 weeks on tofacitinib $10 \mathrm{mg}$ twice daily and subsequently received tofacitinib $5 \mathrm{mg}$ twice daily in the OPEN study. Based on the time-point in the SUSTAIN trial that remission state was achieved, the patients were further divided into 3 groups. Patients who were in remission at baseline in the SUSTAIN trial were included in the first group (remission $\geq 12$ months pre-dose reduction); patients in remission at 24 weeks were included in the second group (remission $6-<12$ months pre-dose reduction); and patients in remission at 52 weeks were included in the third group (remission $<6$ months pre-dose reduction). Overall, rates for maintenance of remission were $82 \%$ at 12 months and $76 \%$ at 24 months in the OPEN study. Among the 3 different groups, the remission rates at 12 months were $91 \%$ for the first group, $82 \%$ for the second group, and $71 \%$ for the third group. This data suggest that the majority of UC patients who achieved stable remission with tofacitinib $10 \mathrm{mg}$ twice daily and dose reduced to $5 \mathrm{mg}$ twice daily maintained remission for up to 24 months 
following the dose reduction, although the authors highlighted that this benefit may be greater in those patients who are in remission for $\geq 6$ months prior to dose reduction.

\section{Effect on Health-Related Quality of Life (HRQoL)}

The effect of tofacitinib on HRQoL was investigated across the single phase II, the two OCTAVE Induction and the single OCTAVE Sustain trials. This was performed using the disease-specific Inflammatory Bowel Disease Questionnaire (IBDQ) in the phase II trial, and both the IBDQ and generic SF-36v2 ${ }^{\circledR}$ Health Survey (SF-36) in the other trials.

In the phase II trial, IBDQ was completed at baseline (day 1), with total scores being similar across all treatment groups, ranging from 123.2 to $134.5 .^{52}$ At week 8, mean IBDQ total scores had dose-proportionally improved compared to baseline measurements across all five treatment groups (week 8 range 149.6-175.4), with the improvement being significantly higher over placebo $(p=0.001)$ only for those patients who received tofacitinib $15 \mathrm{mg}$ twice daily. Patients in endoscopic remission were found to have significantly higher IBDQ scores than those who had not achieved endoscopic remission.

In the two induction trials, IBDQ was completed at baseline (day 1), week 4, and week 8 , whereas SF-36 was completed at baseline and week $8 .^{53}$ At week 8 , mean changes from baseline IBDQ were higher in those patients treated with tofacitinib $10 \mathrm{mg}$ twice daily (40.7 and 44.6) compared to those receiving placebo (21.0 and 25.0, $\mathrm{p}<0.0001)$. Similar findings were observed for SF-36, which measures mean changes from baseline in Physical and Mental Component Summaries (PCS/MCS). The
OCTAVE induction 1 and 2 trials show PCM 6.8 and 6.8, and MCS 6.8 and 7.6, respectively, for those patients receiving tofacitinib $10 \mathrm{mg}$ vs PCS 2.5 and 4.6 and MCS 3.5 and 4.4 for those receiving placebo $(\mathrm{p}<0.01)$. In both trials, HRQoL was found to be significantly correlated with clinical remission and mucosal healing. In the Sustain trial, IBDQ was completed at weeks 8, 16, 24, 32, 40, and 52, whereas SF-36 was completed at weeks 24 and 52. At week 52, changes from baseline in IBDQ total scores were maintained with tofacitinib (3.7 for those receiving $5 \mathrm{mg}$ twice daily and 4.8 for those at $10 \mathrm{mg}$ ) as compared to placebo $(-26.5, \mathrm{p}<0.0001)$. The conclusion for the observed changes in the PCS $(0,0.3$ for tofacitinib 5 and $10 \mathrm{mg}$ twice daily vs -5.2 for placebo, $\mathrm{p}<0.0001)$ and MCS subscores $(-1,0.1$ for tofacitinib 5 and $10 \mathrm{mg}$ vs -6.7 for placebo, $\mathrm{p}<0.0001$ ) of SF-36 was similar.

Overall, tofacitinib was found to result in significant improvements in HRQoL compared to placebo, and this was strongly related to clinical and endoscopic remission. Similar conclusions were reached when data from the induction and maintenance trials were re-analyzed, using the comparatively more stringent IBDQ criteria for remission (total score $\geq 190$ ) and response ( $\geq 30$-point increase from baseline). ${ }^{50}$

\section{Real-World Experience}

The real-world experience of tofacitinib in the management of active UC derives from three retrospective studies from tertiary referral centers across USA and France (Table 2). Weisshof et al describe their experience of tofacitinib in a cohort of 58 patients with inflammatory bowel disease with UC, 4 with Crohn's disease, 1 with

Table 2 Real-World Studies

\begin{tabular}{|c|c|c|c|c|}
\hline & Number & Previous Biologics & Dose & Outcomes \\
\hline Weisshof et al (5I) & $\begin{array}{l}58 \text { patients } \\
\text { (53 with UC) }\end{array}$ & $\begin{array}{l}93 \% \text { failed } \mathrm{TNFi} \\
81 \% \text { failed VDZ }\end{array}$ & $\begin{array}{l}60 \%(n=30) \text { received } 10 \mathrm{mg} \text { BID } \\
38 \%(n=22) \text { received } 5 \mathrm{mg} \text { BID } \\
2 \%(n=1) \text { received II Img ER OD }\end{array}$ & $\begin{array}{l}\text { Week } 8 \text { clinical response } 69 \% \text {, clinical } \\
\text { remission } 33 \% \text {, and corticosteroid-free } \\
\text { remission } 26 \% \\
\text { Week } 52 \text { corticosteroid-free remission } 27 \%\end{array}$ \\
\hline Ungaro et al (52) & $\begin{array}{l}\text { I } 23 \text { patients } \\
\text { with UC }\end{array}$ & $\begin{array}{l}28.5 \% \text { biologic naïve } \\
40.7 \% \text { failed both } \\
\text { TNFi \& VDZ }\end{array}$ & All received IOmg BID & $\begin{array}{l}\text { Week } 8 \text { clinical response } 60.8 \% \text { and } \\
\text { clinical remission } 13.5 \% \\
\text { Week } 16 \text { response } 55.4 \% \text {, remission } \\
48.6 \%(n=74) \\
\text { Month } 6 \text { endoscopic healing } 64.9 \%(n=57)\end{array}$ \\
\hline $\begin{array}{l}\text { Lair-Mehiri et al } \\
\text { (53) }\end{array}$ & $\begin{array}{l}37 \text { patients } \\
\text { with UC }\end{array}$ & $\begin{array}{l}\text { All failed TNFi } \\
97 \% \text { failed VDZ }\end{array}$ & All received $10 \mathrm{mg}$ BID & $\begin{array}{l}\text { Week } 24 \text { colectomy-free survival } 77 \% \text {, } \\
\text { clinical response } 41 \% \text {, and corticosteroid- } \\
\text { free remission } 32 \%\end{array}$ \\
\hline
\end{tabular}


pouchitis). ${ }^{51}$ The majority (93\%) of the patients had previously failed treatment with anti-TNF $\alpha$ and $81 \%$ had failed treatment with vedolizumab, thus constituting a highly medically refractory population. Tofacitinib dose was selected based on symptom severity, with $35 / 58$ $(60 \%)$ receiving tofacitinib $10 \mathrm{mg}$ twice daily, 22/58 (38\%) receiving tofacitinib $5 \mathrm{mg}$ twice daily, and one patient receiving an extended-release tofacitinib $10 \mathrm{mg}$ once daily. Of those receiving $10 \mathrm{mg}$ twice daily, 7 patients started on tofacitinib $15 \mathrm{mg}$ twice daily for severe disease before reducing to $10 \mathrm{mg}$ twice daily within 2-4 weeks due to limited efficacy. Of those receiving $5 \mathrm{mg}$ twice daily, 10 patients were escalated to $10 \mathrm{mg}$ twice daily, with 8 achieving clinical response. All patients received tofacitinib for at least 8 weeks and clinical response (defined as symptomatic improvement from baseline) was assessed at 8,26 , and 52 weeks. After 8 weeks of treatment, 21 patients $(36 \%)$ achieved a clinical response and $19(33 \%)$ achieved clinical remission, corresponding to an overall response rate of $69 \%$. The proportion of patients still being maintained on tofacitinib at 3, 6, and 12 months was $69 \%, 55 \%$, and $51 \%$, respectively. Corticosteroid-free remission occurred in $26 \%, 21 \%$, and $27 \%$ of the patients at 8,26 , and 52 weeks, respectively.

In another cohort study from six centers in the USA, Ungaro et al retrospectively reviewed the data of 123 patients with active UC who received tofacitinib $10 \mathrm{mg}$ twice daily for at least 8 weeks. ${ }^{52}$ A minority (28.5\%) of patients were biologic naïve, whilst $40.7 \%$ had been exposed to both anti-TNF $\alpha$ and VDZ. Clinical response (defined as $>50 \%$ reduction in symptoms) and clinical remission (defined as absence of symptoms) was assessed at week 8 and week 16, and endoscopic healing (defined as Mayo endoscopic score $\leq 1$ or absence of erosions/ulcerations) was assessed within 6 months of initiating tofacitinib. Ninety-six patients completed 8 weeks of treatment with an overall clinical response of $60.8 \%$ and clinical remission of $13.5 \%$. At week 16, 74 patients remained on tofacitinib, with a clinical response of $55.4 \%$ and clinical remission of $48.6 \%$. The majority of patients $(64.9 \%$, $\mathrm{n}=57)$ achieved endoscopic healing at 6 months. Subgroup analysis showed that those patients naive to biologics responded significantly better to tofacitinib than those with previous exposure. The patient's naïve status (adjusted odds ratio [aOR] 5.50, 95\% CI 1.71-17.65), the concomitant use of corticosteroids (aOR 0.25, 95\% CI $0.07-0.83$ ) and male gender (adjusted hazard ratio 0.25 ,
95\% CI 0.08-0.83) were found to be independently associated with clinical response at week 8 .

A French multi-center retrospective study included 37 patients with medically refractory UC treated with tofacitinib $10 \mathrm{mg}$ twice daily for at least 24 weeks. ${ }^{53}$ All patients had previously been treated with a TNFi and 97\% with VDZ. The primary endpoint was colectomy-free survival rate at week 24 and was achieved in $77 \%$ of the patients. Survival without drug interruption was seen in 25 patients $(62.6 \%, 44.3-76.4 \%)$ at week 24 and 9 patients at week 48 $(58.2 \%, 39.2-73.1 \%)$. At 24 weeks, clinical response was achieved by $41 \%$ of the patients, with $32 \%$ being in a corticosteroid-free clinical remission status.

Collectively, these three studies show that tofacitinib is an effective treatment in the management of refractory UC in a real-world setting.

\section{Comparative Efficacy of Tofacitinib vs Biological Therapies}

In the absence of head-to-head trials, evidence of the comparative efficacy of tofacitinib and biological therapies is based on indirect evidence from four network metaanalyses (NMA). These all compared tofacitinib with antiTNF $\alpha$ (infliximab, adalimumab, golimumab) and antiintegrins (VDZ), and all synthesized data from randomized controlled phase II and/or III trials. Three out of four NMAs used clinical (response and remission) and endoscopic (MH) efficacy endpoints as outcomes, ${ }^{54-56}$ whereas one used HRQoL. ${ }^{57}$

Singh and Bonovas undertook their analyses of clinical and endoscopic endpoints in anti-TNF $\alpha$ naïve cohorts by basing their probabilities of each treatment being superior. The authors concluded that infliximab and VDZ may be superior for the induction of clinical response, remission, and $\mathrm{MH}^{58,59}$ Trigo-Vicente et al reached similar conclusions. $^{56}$ However, the addition of ozanimod (a sphingosine-1-phosphate receptor agonist) and etrolizumab (an anti-adhesion molecule antibody) in their analysis showed that ozanimod may be better than infliximab for the achievement of $\mathrm{MH}$. In contrast, for patients who were anti-TNF $\alpha$ experienced, tofacitinib was ranked higher for the induction of remission and of $\mathrm{MH}$ compared to the other available treatments. ${ }^{58}$ For the maintenance of remission, no indirect comparisons were performed for antiTNF $\alpha$ experienced patients. ${ }^{54-56}$ However, in anti-TNF $\alpha$ naïve patients, Trigo-Valente et al found that tofacitinib had the highest probability of being the best treatment in 
the maintenance of remission, sustained remission and for $\mathrm{MH}$.

With regard to the impact of different therapies on HRQoL, at induction, Paschos et al found that all of the compared therapies significantly improved both generic and disease-specific HRQL scores compared to placebo. ${ }^{57}$ However, the improvement was clinically meaningful only for infliximab (18.58; 95\% CI $13.19-$ 23.97) and VDZ (18.00; 95\% CI 11.08-24.92). For maintenance, VDZ, tofacitinib, and adalimumab were found to maintain the improvement in HRQL.

\section{Safety of Tofacitinib in UC}

The first centralized evaluation of the short- and long-term safety profile of tofacitinib in the management UC was recently published. ${ }^{58}$ The report was focused on specific adverse events (AEs), including herpes zoster (HZ), opportunistic infections (OIs), malignancies, non-melanoma skin cancers (NMSC), major adverse cardiovascular events (MACE), and gastrointestinal perforations. The safety data were collated from the four aforementioned market-authorizing trials, including 1157 patients over a 4.4-year period (1612.8 patient-years of exposure). They were analyzed as 3 cohorts: the induction cohort, which included data from patients randomized to placebo or tofacitinib from the dose-finding phase II and the two phase III induction trials; the maintenance cohort, that included data from patients from the Sustain trial; and the laboratory cohort that included data from patients from the induction and the sustain cohort who were evaluated for changes over time in laboratory parameters. Serious adverse events (SAEs) were defined as any events threatening life, resulting in death, causing persistent or significant disability or incapacity, requiring or prolonging hospitalization, or causing congenital anomaly or birth defect.

In the induction cohort, $54.9 \%$ of the patients developed AEs and 3.8\% developed SAEs resulting in tofacitinib being stopped. Similar rates were seen in patients receiving placebo, with AEs in $55.0 \%$ and SAEs in $5.0 \%$. In contrast, higher rates of AEs $(75.3 \%, 72.2 \%$, and $79.6 \%$ for those receiving placebo, tofacitinib $5 \mathrm{mg}$, and $10 \mathrm{mg}$ twice daily, respectively) and of SAEs $(6.6 \%$, $5.1 \%$, and $5.6 \%$ ) were found across all of the treatment arms in the maintenance cohort.

The most frequent adverse events seen in the induction cohort were headache $(6.7 \%$ for placebo, $7.8 \%$ for tofacitinib $10 \mathrm{mg}$ ) and nasopharyngitis (5.0\% for placebo, 6.0\% for tofacitinib $10 \mathrm{mg}$ ), whereas nasopharyngitis $(5.6 \%$ for placebo, $9.6 \%$ for tofacitinib $5 \mathrm{mg}, 13.8 \%$ for tofacitinib $10 \mathrm{mg}$ ) and worsening of UC (35.9\% for placebo, $18.2 \%$ for tofacitinib $5 \mathrm{mg}, 14.8 \%$ for tofacitinib $10 \mathrm{mg}$ ) prevailed in the maintenance cohort. Serious infections (SI), defined as any treated infections that required parenteral antimicrobial therapy, hospitalization, or fulfilled criteria for SAEs, were rarely observed (IR: 2.0; 95\% CI: 1.4-2.8). However, there were four types of SI that were reported more than once including appendicitis, herpes zoster infection (HZ), anal abscess, and Clostridium difficile infection. None of these infections were fatal.

In the maintenance cohort, the incidence ratio for $\mathrm{HZ}$ was found to be increased in UC, with greater values being observed in those patients receiving tofacitinib $10 \mathrm{mg}$ compared to those receiving tofacitinib $5 \mathrm{mg}$ or placebo. This finding signified a dose-proportional increase of the associated risk for HZ. In the overall cohort, there were recorded 18 cases of $\mathrm{HZ}$, with older age, prior anti-TNF $\alpha$ failure, and non-white (mainly Asians) race being independently associated with an increased risk for developing HZ. However, in most cases, HZ infection was cutaneous over 1 or 2 adjacent dermatomes and did not require permanent discontinuation of tofacitinib. A more detailed analysis of $\mathrm{HZ}$ events in the UC program has been reported by Winthrop et al. ${ }^{59}$ In this study, based on their previous experience in RA, the authors suggested that vaccination against $\mathrm{HZ}$ could be a possible preventive strategy for nonexposed UC patients; however, in the absence of robust data from UC, vaccination is not currently recommended. An ongoing randomized controlled trial evaluating the safety and immunogenicity of $\mathrm{HZ}$ vaccine across a variety of immune-mediated disorders, including patients with UC (VERVE trial, NCT02538341), ${ }^{60}$ is expected to provide some more definitive results on this.

During the observation period in the OCTAVE trials, four deaths were recorded in the overall population, with 3 out of 4 cases being secondary to malignancies (hepatic angiosarcoma, acute myeloid leukemia, and cholangiocarcinoma). In the overall cohort, 22 patients were diagnosed with malignancy, with $50 \%$ of the cases having NMSC. The majority (18 out of the 22) of patients were experienced with anti-TNF $\alpha$ and thiopurines, whereas 6 out of the 11 with NMSC had previous history of NMSC. Overall, malignancies were rarely observed.

With regard to other adverse events, 3 cases of colonic perforations and 4 MACEs (hemorrhagic stroke, aortic dissection, acute coronary syndrome, and myocardial 
infarction) were recorded in the study. Nearly all of the involved (5/7) patients had multiple risk factors that may have contributed to the development of these complications. In particular, 2 out of the 3 cases of perforation occurred in patients with a background of active UC inflammation or Epstein-Barr virus intestinal lymphoma who were recently prescribed corticosteroids and underwent an endoscopic procedure, whereas the third case occurred in a patient who developed appendicitis and received concomitant nonsteroidal anti-inflammatory drugs. Likewise, 3 out of 4 with MACE had $\geq 4$ predisposing cardiovascular risk factors. However, other than a case of aortic dissection that resulted in death, all of the other MACE were resolved after temporary or permanent discontinuation of TOF.

No clinically meaningful changes were observed in various laboratory parameters, including the low-density lipoprotein/high-density lipoprotein ratio, hemoglobin, absolute lymphocyte count, and creatine kinase. ${ }^{61}$

An update of the results of this study was presented by Sandborn et al in the most recent ECCO 2019 conference, extending our knowledge on the safety profile of tofacitinib by 1 year. ${ }^{62}$ No additional or unexpected safety signals were identified, supporting the long-term use of tofacitinib in patients with moderately to severely active UC.

\section{Safety of Tofacitinib vs Biological Therapies}

As with comparisons of the efficacy between tofacitinib and biological therapy, comparative safety data are derived indirectly from NMAs. Trigo-Vicente et al showed that all comparative treatments were more likely to cause SAEs than placebo and that all therapies had similar probabilities of causing SAEs. ${ }^{60}$ With regards to opportunistic infections, tofacitinib had the highest rate (although most were minor), whilst golimumab and vedolizumab also showed increased rates, and infliximab and adalimumab did not show a statistically significant increased rate compared to placebo.

\section{Safety of Tofacitinib in Specific Populations}

The safety of tofacitinib in children, pregnant women, and elder patients has not been directly studied in UC. However, Mahadevan et al recently reported pregnancy and infant outcomes from patients with both maternal and paternal prenatal exposure to tofacitinib who participated in the five aforementioned UC clinical trials. ${ }^{63}$ Of the 1157 patients enrolled in these studies, 301 were women of childbearing age with 11 cases of maternal exposure and 14 cases of paternal exposure identified. In total, they reported 15 healthy newborns, no fetal or neonatal deaths, no congenital malformations, 2 spontaneous abortions, and 2 medical terminations. These findings appear similar to those reported from other clinical study populations and the general population. ${ }^{64,65}$ Nevertheless, more data are clearly needed to evaluate safety in this particular setting. ${ }^{67-69}$

\section{Safety of Tofacitinib in Other Disease Populations}

The incidence ratio of adverse events of special interest in the tofacitinib UC program was generally consistent with those reported in the RA program. However, a postmarketing study (A3921133) in RA found an increased incidence of pulmonary embolism among patients over 50 years with more than 1 cardiovascular factor receiving the $10 \mathrm{mg}$ twice daily dose. This finding has led regulatory authorities to restrict the use of tofacitinib in patients with an increased risk for thromboembolism, which includes heart failure, cancer, thrombophilia, previous history of thromboembolic episodes, patients receiving hormonal contraceptives or hormone replacement therapy and those undergoing major surgery. ${ }^{66}$ An ongoing safety trial in RA and future post-marketing studies in UC are expected to shed more light into this relationship.

\section{Potential Place in UC Pharmacotherapy}

Tofacitinib is the first oral JAK inhibitor that has been approved by the EMA and the FDA for the management of adult patients with moderately to severely active UC. The American College of Gastroenterology (ACG) has recently positioned tofacitinib in the therapeutic algorithm of UC either as first-line therapy for moderate-to-severely active disease or as second-line therapy in those who have failed anti-TNF $\alpha .{ }^{67}$ In addition, the real-world studies described above show that tofacitinib can be used effectively as third-line therapy in patients who have failed both anti$\mathrm{TNF} \alpha$ and vedolizumab. Although the NMAs suggest tofacitinib may be less effective than anti-TNF $\alpha$ as a firstline therapy and more effective as a second-line therapy, more substantial evidence is needed to inform selection preference. 
As tofacitinib is the latest therapy to be approved for $\mathrm{UC}$, its current use mostly assumes that of salvage therapy. However, compared to the available biologics (infliximab, adalimumab, golimumab, and VDZ), tofacitinib has several potentially favorable characteristics that could lead to its preferential selection. Firstly, it is orally administered which could improve therapy adherence and quality of life in patients averse to the requirement for long-term injections or infusions. ${ }^{68,69}$ Its pharmacokinetic profile, characterized by rapid absorption, metabolism, and clearance, is advantageous in situations where rapid onset of action (e.g., acute severe colitis) or elimination of the drug (e.g., infection, surgery, or pregnancy) is required. ${ }^{70}$ Importantly, tofacitinib is a small synthetic molecule that would not induce an immunogenic response. Therefore, unlike the anti-TNF $\alpha$ in particular, there is no risk of loss of response due to antibody formation against the drug, negating the need for combination therapy with an immunomodulator to prevent this. ${ }^{35}$ In addition, tofacitinib would be expected to have lower costs than the biologics, given it is a small molecule with less elaborate production and will therefore have a higher cost-effectiveness ratio. ${ }^{71-73}$

\section{Future Directions}

The evidence on the efficacy and safety of tofacitinib for the management of UC mainly arises from clinical trials and pertain to restricted populations. Post-marketing studies are needed to expand clinicians' experience on the efficacy and safety of tofacitinib in real-world populations, including specific groups excluded from the licensing trials (e.g., elders and children), and to address the potential thromboembolic risk highlighted in the RA population.

With the approval of tofacitinib for the management of moderately-to-severely active UC, the available therapeutic options have increased, creating an issue with selection. Current evidence is insufficient to support preferential selection amongst the available therapeutic options and direct head-to-head comparison trials are necessary to address this issue. ${ }^{74}$

\section{Conclusion}

Tofacitinib is a valuable addition to the therapeutic armamentarium for managing UC. The OCTAVE trials have shown that tofacitinib is effective for the management of moderately-to-severely active UC with a good overall tolerance, although the potential risk of thromboembolism seen in the RA population needs further evaluation. Current recommendations issued by the ACG have positioned tofacitinib alongside the established biologic agents for both induction and maintenance of remission of moderate-to-severely active UC. Although tofacitinib possesses several favorable characteristics (e.g., oral administration, lack of immunogenicity, rapid action, and clearance), the exact place against more established biological therapies will become more evident as clinical experience with this novel agent improves.

\section{Disclosure}

Dr Argyriou has received a grant from the Hellenic Society of Gastroenterology for post-doctoral research at Nottingham Digestive Diseases Centre (protocol number: 7406/20-12-2016). Dr Varyani has received speaker honoraria from Takeda Pharmaceuticals and has received educational support from Takeda and Abbvie. Dr Moran has received educational support from Abbvie, Janssen, NAPP, Takeda Pharmaceuticals, Merck Sharp \& Dohme Ltd, Ferring, and Dr Falk. He has received speaker honoraria from Merck Sharp \& Dohme Ltd, Abbvie, Janssen, Ferring and Takeda Pharmaceuticals. He attended advisory boards for Abbvie, Takeda Pharmaceuticals, Janssen, Medtronic, Phebra Pharmaceuticals, Servertus Associates Ltd and Dr Falk. The authors report no other conflicts of interest in this work.

\section{References}

1. Ungaro R, Mehandru S, Allen PB, Peyrin-Biroulet L, Colombel JF. Ulcerative colitis. Lancet. 2017;389:1756-1770. doi:10.1016/S01406736(16)32126-2

2. Burisch J, Munkholm P. The epidemiology of inflammatory bowel disease. Scand J Gastroenterol. 2015;50(8):942-951. doi:10.3109/ 00365521.20 15.1014407

3. Molodecky NA, Soon I, Rabi DM, et al. Increasing incidence and prevalence of the inflammatory bowel diseases with time, based on systematic review. Gastroenterology. 2012;421:46-54. doi:10.1053/j. gastro.2011.10.001

4. Hoivik ML, Solberg IC, Henriksen M, Cvancarova M, Bernklev T. Work disability in inflammatory bowel disease patients 10 years after disease onset: results from the IBSEN Study. Gut. 2013;62:368-375. doi:10.1136/gutjnl-2012-302311

5. Fumery M, Singh S, Dulai PS, Gower-Rousseau C, Peyrin-Biroulet L, Sandborn WJ. Natural history of adult ulcerative colitis in population-based cohorts: a systematic review. Clin Gastroenterol Hepatol. 2018;16:343-356. doi:10.1016/j.cgh.2017.06.016

6. Solberg IC, Lygren I, Jahnsen J, et al. Clinical course during the first 10 years of ulcerative colitis: results from a population-based inception cohort (IBSEN Study). Scand J Gastroenterol. 2009;44:431-440. doi:10.1080/00365520802600961

7. Magro F, Gionchetti P, Eliakim R, et al. Third European evidence-based consensus on diagnosis and management of ulcerative colitis. Part 1: definitions, diagnosis, extra-intestinal manifestations, pregnancy, cancer surveillance, surgery, and ileo-anal pouch disorders. J Crohns Colitis. 2017;11(6):649-670. 
8. Sandborn WJ, Feagan BG, Marano C, et al. Subcutaneous golimumab maintains clinical response in patients with moderate-to-severe ulcerative colitis. (1528-0012 (Electronic)). Gastroenterology;146(1):96-109.

9. Sandborn WJ, van Assche G, Reinisch W, et al. Adalimumab induces and maintains clinical remission in patients with moderate-to-severe ulcerative colitis. Gastroenterology. 2012;142:257-265. doi:10.1053/ j.gastro.2011.10.032

10. Reinisch W, Sandborn WJ, Hommes DW, et al. Adalimumab for induction of clinical remission in moderately to severely active ulcerative colitis: results of a randomised controlled trial. Gut. 2011;60:780-787. doi:10.1136/gut.2010.221127

11. Rutgeerts P, Sandborn WJ, Feagan BG, et al. Infliximab for induction and maintenance therapy for ulcerative colitis. $N$ Engl $\mathrm{J} \mathrm{Med}$. 2005;353: 2462-2476. doi:10.1056/NEJMoa050516

12. Neurath MF. Cytokines in inflammatory bowel disease. Nat Rev Immunol. 2014;14:329-342. doi:10.1038/nri3661

13. Fujino S, Andoh A, Bamba S, et al. Increased expression of interleukin 17 in inflammatory bowel disease. Gut. 2003 52: 65-70. doi:10.1136/gut.52.1.65

14. Kulkarni AB, Huh CG, Becker D, et al. Transforming growth factor beta 1 null mutation in mice causes excessive inflammatory response and early death. Proc Natl Acad Sci U S A. 1993;15:770-774. doi:10.1073/pnas.90.2.770

15. Shouval DS, Biswas A, Goettel JA, et al. Interleukin-10 receptor signaling in innate immune cells regulates mucosal immune tolerance and anti-inflammatory macrophage function. Immunity. 2014;40: 706-719. doi:10.1016/j.immuni.2014.03.011

16. Dowty ME, Lin J, Ryder TF, et al. The pharmacokinetics, metabolism, and clearance mechanisms of tofacitinib, a janus kinase inhibitor, in humans. Drug Metab Dispos. 2014;42:759-773. doi:10.1124/ dmd.113.054940

17. Soendergaard C, Bergenheim FH, Bjerrum JT, Nielsen OH. Targeting JAK-STAT signal transduction in IBD. Pharmacol Ther. 2018;192:100-111. doi:10.1016/j.pharmthera.2018.07.003

18. FDA. 2019. Available from: https:/www.accessdata.fda.gov/drug satfda_docs/label/2018/203214s018lbl.pdf.

19. Pfizer. EU summary of product characteristics 2019. Available from: https://www.ema.europa.eu/en/documents/variation-report/xeljanzh-c-4214-x-0005-epar-assessment-report-variation_en.pdf.

20. XR XX. Highlights of prescribing information; 2019. Available from: https://www.accessdata.fda.gov/drugsatfda_docs/label/2018/ 203214s018lbl.pdf.

21. Yamaoka K, Saharinen P, Pesu M, Holt VET 3rd, Silvennoinen O, O'Shea JJ. The Janus kinases (Jaks). Genome Biol. 2004;5:253. doi:10.1186/gb-2004-5-12-253

22. Banerjee S, Biehl A, Gadina M, Hasni S, Schwartz DM. JAK-STAT signaling as a target for inflammatory and autoimmune diseases: current and future prospects. Drugs. 2017;77:521-546. doi:10.1007/ s40265-017-0701-9

23. O'Shea JJ, Holland SM, Staudt LM. JAKs and STATs in immunity, immunodeficiency, and cancer. N Engl J Med. 2013;368:161-170. doi:10.1056/NEJMra1202117

24. Can G, Tezel A, Gurkan H, et al. Tyrosine kinase-2 gene polymorphisms are associated with ulcerative colitis and Crohn's disease in Turkish Population. Clin Res Hepatol Gastroenterol. 2015;39: 489-498. doi:10.1016/j.clinre.2015.01.005

25. Aggarwal BB. Signalling pathways of the TNF superfamily: a double-edged sword. Nat Rev Immunol. 2003;3(9):745-756. doi:10. 1038/nri1184

26. Rovira J, Ramirez-Bajo MJ, Banon-Maneus E, et al. Tofacitinib halts progression of graft dysfunction in a rat model of mixed cellular and humoral rejection. Transplantation. 2018;102:1075-1084. doi:10.10 97/TP.0000000000002204

27. Changelian PS, Flanagan ME, Ball DJ, et al. Prevention of organ allograft rejection by a specific Janus kinase 3 inhibitor. Science. 2003;302:875-878. doi:10.1126/science.1087061
28. Ghoreschi K, Jesson MiLi X, Lee JL, et al. Modulation of innate and adaptive immune responses by tofacitinib (CP-690,550). J Immunol. 2011;86:4234-4243.

29. Boland BS, Sandborn WJ, Chang JT. Update on Janus kinase antagonists in inflammatory bowel disease. Gastroenterol Clin North Am. 2014;43:603-617. doi:10.1016/j.gtc.2014.05.011

30. Levy DE, Darnell JE Jr. Stats: transcriptional control and biological impact. Nat Rev Mol Cell Biol. 2002;3(9):651-62 doi:10.1038/ nrm909

31. Hodge JA, Kawabata TT, Krishnaswami S, et al. The mechanism of action of tofacitinib - an oral Janus kinase inhibitor for the treatment of rheumatoid arthritis. Clin Exp Rheumatol. 2016;34:318-328.

32. Meyer DM, Jesson MI, Li X, et al. Anti-inflammatory activity and neutrophil reductions mediated by the JAK1/JAK3 inhibitor, CP-690,550, in rat adjuvant-induced arthritis. $J$ Inflamm (Lond). 2010;7:1476-9255 (Electronic).

33. Krishnaswami S, Boy M, Chow V, Chan G. Safety, tolerability, and pharmacokinetics of single oral doses of tofacitinib, a Janus kinase inhibitor, in healthy volunteers. Clin Pharmacol Drug Dev. 2015; 4:83-88. doi:10.1002/cpdd.171

34. Mukherjee A, Hazra A, Smith MK, et al. Exposure-response characterization of tofacitinib efficacy in moderate to severe ulcerative colitis: results from a dose-ranging phase 2 trial. $\mathrm{Br} J \mathrm{Clin}$ Pharmacol. 2018;84:1136-1145. doi:10.1111/bcp.13523

35. Ma G, Xie R, Strober B, et al. Pharmacokinetic characteristics of tofacitinib in adult patients with moderate to severe chronic plaque psoriasis. Clin Pharmacol Drug Dev. 2018;7: 587-596. doi:10.1002/ cpdd. 471

36. Gupta P, Alvey C, Wang R, et al. Lack of effect of tofacitinib (CP-690,550) on the pharmacokinetics of the CYP3A4 substrate midazolam in healthy volunteers: confirmation of in vitro data. $\mathrm{Br} J \mathrm{Clin}$ Pharmacol. 2012;74:109-115. doi:10.1111/bcp.2012.74.issue-1

37. Gupta P, Chow V, Wang R, et al. Evaluation of the effect of fluconazole and ketoconazole on the pharmacokinetics of tofacitinib in healthy adult subjects. Clin Pharmacol Drug Dev. 2014;3:72-77. doi:10.1002/cpdd.v3.1

38. Sandborn WJ, Ghosh S, Panes J, et al. Tofacitinib, an oral Janus kinase inhibitor, in active ulcerative colitis. $N$ Engl $\mathrm{J} \mathrm{Med}$. 2012;367:616-624. doi:10.1056/NEJMoa1112168

39. Kremer JM, Bloom BJ, Breedveld FC, et al. The safety and efficacy of a JAK inhibitor in patients with active rheumatoid arthritis: results of a double-blind, placebo-controlled phase IIa trial of three dosage levels of CP-690,550 versus placebo. Arthritis Rheum. 2009;60: 1895-1905. doi:10.1002/art.24567

40. Sandborn WJ, Su C, Sands BE, et al. Tofacitinib as induction and maintenance therapy for ulcerative colitis. $N$ Engl J Med. 2017;376:1723-1736. doi:10.1056/NEJMoa1606910

41. Motoya S, Watanabe M, Kim HJ. et al. Tofacitinib induction and maintenance therapy in East Asian patients with active ulcerative colitis: subgroup analyses from three phase 3 multinational studies. Intest Res;2018. 233-245. doi:10.5217/ir.2018.16.2.233

42. Hanauer S, Panaccione R, Danese S, et al. Tofacitinib induction therapy reduces symptoms within 3 days for patients with ulcerative colitis. Clin Gastroenterol Hepatol. 2019;17: 139-147. doi:10.1016/j. cgh.2018.07.009

43. Hanauer S, Rubin DT, Gionchetti P. Tofacitinib efficacy in patients with moderate to severe ulcerative colitis: subgroup analyses of OCTAVE induction 1 and 2 and OCTAVE Sustain by 5-aminosalicylates use. Journal of Crohn's and Colitis. 2019;13 (Supplement_1):S477.doi:10.1093/ecco-jcc/jjy222.836

44. Reinisch W, Osterman MT, Doherty G, et al. . Efficacy of Tofacitinib Maintenance Therapy for Ulcerative Colitis in Remitting Patients Vs. patients with Clinical Response After 8 Weeks of Induction Treatment. Journal of Crohn's and Colitis. 2019;13 (Supplement_1):S378S379. doi:10.1093/ecco-jcc/jjy222.655 
45. Panes J, Bressler B, Colombel JF, et al. Editor Efficacy and safety of tofacitinib retreatment for ulcerative colitis after treatment interruption: results form the octave clinical trials. Digestive diseases week. Gastroenterology. 2019;154.

46. Sands BE, Moss AC, Armuzzi A. Efficacy and safety of dose escalation to tofacitinib $10 \mathrm{mg}$ BID for patients with ulcerative colitis following loss of response on tofacitinib $5 \mathrm{mg}$ BID maintenance therapy: results form OCTAVE open. Digestive Dis Week. 2018; 154:S-385.

47. Rubin DT, Dubinsky MC, Lukas M et al. Long-term efficacy of tofacitinib in patients who received extended induction therapy: results of the OCTAVE open study for tofacitinib delayed responders. Journal of Crohn's and Colitis. 2019;13 (Supplement_1):S050-S052. doi:10.1093/ecco-jcc/jjy222.077

48. Chiorean M, Su C, Matsuoka $\mathrm{K}$ et al. Efficacy and safety of open-label treatment with tofacitinib $10 \mathrm{mg}$ twice daily in patient with ulcerative colitis with clinical response, but not remission, after 52 weeks of maintenance therapy: data from the OCTAVE studies. Journal of Crohn's and Colitis, 2019;13 (Supplement_1): S049-S050. doi:10.1093/ecco-jcc/jjy222.075

49. Rubin DT, Travis S, Abraham BP, et al. Maintenance of efficacy following tofacitinib dose reduction in patients with ulcerative colitis in stable remission. .Journal of Crohn's and Colitis. 2019;13 (Supplement_1): S425-S426. doi:10.1093/ecco-jcc/jjy222.743

50. Dubinsky $\overline{M C}$, Bressler B, Armuzzi A et al. Improvement in patient-reported Inflammatory Bowel Disease Questionnaire outcomes, and relationship with disease activity, in tofacitinib treated patient with ulcerative colitis: data form the OCTAVE clinical trials. Journal of Crohn's and Colitis. 2019;13 (Supplement_1):S462S463. doi:10.1093/ecco-jcc/jjy222.809

51. Weisshof R, Aharoni Golan M, Sossenheimer PH, et al. Real-world experience with tofacitinib in IBD at a tertiary center. Dig Dis Sci. 2019;64:1945-1951. doi:10.1007/s10620-019-05492-y

52. Ungaro R, Fenster M, Dimopoulos C, et al. Real-World effectiveness of tofacitinib in ulcerative colitis: a multi-centre study. Journal of Crohn's and Colitis. 2019;13(Supplement_1):S274S275. doi:10.1093/ecco-jcc/jjy222.468

53. Lair-Mehiri L, Stefanescu C, Vaysse T, et al. Real-world tofacitinib effectivness and safety in patients with refractory ulcerative colitis . Journal of Crohn's and Colitis, 2019; 13 (Supplement_1): S478S479, https://doi.org/10.1093/ecco-jcc/jjy222.839.

54. Mei WQ, Hu HZ, Liu Y, Li ZC, Wang WG. Infliximab is superior to other biological agents for treatment of active ulcerative colitis: a meta-analysis. World J Gastroenterol. 2015;21:6044-6051. doi:10. 3748/wjg.v21.i19.6044

55. Bonovas SA-O, Lytras T, Nikolopoulos G, LA-O P-B, Danese SA-O. Systematic review with network meta-analysis: comparative assessment of tofacitinib and biological therapies for moderate-to-severe ulcerative colitis. Aliment Pharmacol Ther. 2018;47:454-465. doi:10. 1111/apt.14449

56. Trigo-Vicente CA-O, Gimeno-Ballester V, Garcia-Lopez S, LopezDel Val A. Systematic review and network meta-analysis of treatment for moderate-to-severe ulcerative colitis. Int $J$ Clin Pharm. 2018;40:1411-1419. doi:10.1007/s11096-018-0743-4

57. Paschos P, Katsoula A, Giouleme O, et al. Tofacitinib for induction of remission in ulcerative colitis: systematic review and meta-analysis. Ann Gastroenterol. 2018;31:572-582.

58. Sandborn WJ, Panes J, D'Haens GR, et al. Safety of tofacitinib for treatment of ulcerative colitis, based on 4.4 years of data from global clinical trials. Clin Gastroenterol Hepatol. 2019;17(8):1541-1550. doi:10.1016/j.cgh.2018.11.035
59. Winthrop KL, Melmed GY, Vermeire S, et al. Herpes zoster infection in patients with ulcerative colitis receiving tofacitinib. Inflamm Bowel Dis. 2018;24:2258-2265. doi:10.1093/ibd/izy131

60. Curtis J Safety and effectiveness of live zoster vaccine in Anti-Tumor Necrosis Factor (TNF) users (VERVE trial); 2019. Available from: https://clinicaltrials.gov/ct2/show/NCT02538341.

61. Sands BE, Taub PR, Armuzzi A, et al. Tofacitinib treatment is associated with modest and reversible increases in serum lipids in patients with ulcerative colitis. Clin Gastroenterol Hepatol. 2019;30491-4:S1542-S3565. doi:10.1016/j.cgh.2019.04.059

62. Sandborn WJ, Panes J, Panaccione R, et al. Tofacitinib for the treatment of ulcerative colitis: up to 5.4 years of safety data from global clinical trials.Journal of Crohn's and Colitis. 2019;13 (Supplement_1):S344. doi.10.1093/ecco-jcc/jjy222.590

63. Mahadevan $\bar{U}$, Dubinsky MC, Su C, et al. Outcomes of pregnancies with maternal/paternal exposure in the tofacitinib safety databases for ulcerative colitis. Inflamm Bowel Dis. 2018;24:2494-2500. doi:10. 1093/ibd/izy160

64. CDC. Update on overall prevalence of major birth defects-atlanta, Georgia, 1978-2005. MMWR Morb Mortal Wkly Rep. 2008;11:1-5.

65. Clowse MEB, Feldman SR, Isaacs JD, et al. Pregnancy outcomes in the tofacitinib safety databases for rheumatoid arthritis and psoriasis. Drug Saf. 2016;39:755-762. doi:10.1007/s40264-016-0431-z

66. EMA. Restrictions in use of Xeljanz while EMA reviews risk of blood clots in lungs: EMA; Available from: https://www.ema. europa.eu/en/news/restrictions-use-xeljanz-while-ema-reviews-riskblood-clots-lungs.

67. Rubin DT, Ananthakrishnan AN, Siegel CA, Sauer BG, Long MD. ACG clinical guideline: ulcerative colitis in adults. $\mathrm{Am}$ $J$ Gastroenterol. 2019;114:384-413. doi:10.14309/ajg.00000000000 00152

68. Olivera P, Danese S, Peyrin-Biroulet L. JAK inhibition in inflammatory bowel disease. Expert Rev Clin Immunol. 2017;13:693-703. doi:10.1080/1744666X.2017.1291342

69. Olivera P, Danese S, Peyrin-Biroulet L. Next generation of small molecules in inflammatory bowel disease. Gut. 2017;66:199-209. doi:10.1136/gutjnl-2016-312912

70. Berinstein JA, Steiner CA, Regal RE, et al. Efficacy of induction therapy with high-intensity tofacitinib in 4 patients with acute severe ulcerative colitis. Clin Gastroenterol Hepatol. 2019;17:988-990. doi:10.1016/j.cgh.2018.11.022

71. Wu B, Wang Z, Zhang Q. Cost-effectiveness of different strategies for the treatment of moderate-to-severe ulcerative colitis. Inflamm Bowel Dis. 2018;24:2291-2302. doi:10.1093/ibd/izy114

72. Milev S, DiBonaventura MD, Quon P, et al. An economic evaluation of tofacitinib for the treatment of moderately-to-severely active ulcerative colitis: modeling the cost of treatment strategies in the United States. J Med Econ. 2019;22: 859-868. doi:10.1080/ 13696998.2019.1609481

73. Dulai PS, Osterman MT, Lasch K, Cao C, Riaz F, Sandborn WJ. Market access analysis of biologics and small-molecule inhibitors for inflammatory bowel disease among US health insurance policies. Dig Dis Sci. 2019;64:2478-2488. doi:10.1007/s10620-019-05594-7

74. Peyrin-Biroulet L, Lopez A, Sandborn W. Head-to-head comparative studies: challenges and opportunities? J Crohns Colitis. 2017;11: S567-S575. doi:10.1093/ecco-jcc/jjw167 


\section{Publish your work in this journal}

Drug Design, Development and Therapy is an international, peerreviewed open-access journal that spans the spectrum of drug design and development through to clinical applications. Clinical outcomes, patient safety, and programs for the development and effective, safe, and sustained use of medicines are a feature of the journal, which has also

been accepted for indexing on PubMed Central. The manuscript management system is completely online and includes a very quick and fair peer-review system, which is all easy to use. Visit http://www. dovepress.com/testimonials.php to read real quotes from published authors.

Submit your manuscript here: https://www.dovepress.com/drug-design-development-and-therapy-journal 